\title{
Electromagnetic Wave Absorption Properties based on Slot Size of Biomass Hollow Pyramidal Microwave Absorber
}

\author{
Mas Izzati Fazin, Ahmad Rashidy Razali, Hasnain Abdullah@Idris, Mohd Nasir Taib, Norhayati \\ Mohamad Noor, Nazirah Mohamat Kasim, Azizah Ahmad, Linda Mohd Kasim, Noor Azila Ismail
}

\begin{abstract}
Pyramidal microwave absorbers are used in anechoic chambers to eliminate unwanted signal wave that downgrade the quality of electromagnetic wave radiation measurement operation. Microwave absorbers are necessary to eliminate radiation wave reflection and to reduce signal leakage from various electronic equipments. Unfortunately, common material used in cormercial absorbers are often expensive, nonmodifiable and non-biodegradable. To address the problem and hence to achieve optimal electromagnetic wave absorption performance, new investigation into designs of different rectangular slot size on biomass absorber is presented in this paper. Three rectangular slot sizes are considered in the investigation to vary the electromagnetic wave absorption performance. These small, medium and big rectangular slot size are designed on hollow pyramidal microwave absorber are observed to analyzed their absorption performance at the frequency band of $\mathrm{L}, \mathrm{S}, \mathrm{C}$ and $\mathrm{X}$-band. The proposed slotted pyramidal microwave absorber designs are measured using NRL arch free space measurement technique to determine its absorption performance at the frequency bands. Results comparison shows that small slot size posess the highest absorption performance at all frequency bands followed by medium and big slot size. The result indicates that small slot size is a good candidate to improve pyramidal microwave absorber performance.
\end{abstract}

Index Terms - microwave absorber, slot array, hollow pyramidal, electromagnetic wave

\section{INTRODUCTION}

$\mathrm{R}$ ADIO wave and microwave are forms of electromagnetic energy that are collectively described by the term radio frequency (RF). Radio frequencies are divided into groups which have similar characteristics, called "bands" such as Lband, S-band, C-band and X-band. The wavelength of

This manuscript is submitted on $18^{\text {th }}$ January 2021 and accepted on $19^{\text {th }}$ April 2021. Mas Izzati Fazin, Ahmad Rashidy Razali, Hasnain Abdullah@Idris, Norhayati Mohammad Noor, Nazirah Mohamat Kasim, Azizah Ahmad, Linda Mohd Kasim and Noor Azila Ismail are now with the School of Electrical Engineering, Universiti Teknologi MARA cawangan Pulau Pinang, 13500 Permatang Pauh, Penang. (e-mail: mas.ezaty.me@gmail.com, hasnain@uitm.edu.my)

Mohd Nasir Taib is from the School of Electrical Engineering, Universiti Teknologi MARA, 40450 Shah Alam, Selangor

1985-5389/C 2021 The Authors. Published by UiTM Press. This is an open access article under the CC BY-NC-ND license (http://creativecommons.org /licenses/by-nc-nd/4.0/). microwaves are approximately in the range of $30 \mathrm{~cm}(1 \mathrm{GHz})$ to $1 \mathrm{~mm}(300 \mathrm{GHz})$ [1]. Electromagnetic wave travel with a constant velocity of $3 \times 10^{8} \mathrm{~ms}^{-1}$ in vacuum [1], [2]. Moreover, they also can travel through anything either in air, solid material or vacuum. They comply with the laws of reflection and can be transmitted, absorbed or reflected relying on sort of material. Electromagnetic wave vary in wavelength and frequency. All electromagnetic wave propagates at the same speed through space, their wavelengths, frequencies, and energy levels can vary. A wave with longer wavelength have lower frequencies while shorter wavelength will have higher frequencies. The speed of a wave is a product of its wavelength and frequency. Regulation of electromagnetic (EM) disruptions and elimination of radiation field emission are important to avoid interference with electromagnetic and protection problems [3]. Electromagnetic interference (EMI) can cause significant device disruption. Due to system-to-system [4], [5], it can cause computer malfunctions, produce false images and lower efficiency. Electromagnetic wave absorbers with the ability to absorb unwanted electromagnetic waves are used to solve problems caused by electromagnetic interference. New advances in microwave absorber technology have contributed, on the other hand, to materials which can effectively minimize electromagnetic signal reflection and have decent physical quality and lower production costs [6].

In this project, absorbers are designed in pyramidal shape. Excellent performance of pyramidal absorber is primarily due to the many reflections that exist between the pyramids [7], [8]. The multi-reflections in these pyramidal microwave absorbers contribute to substantial attenuation and boost microwave absorption relative to the flat [9]. Incident waves at the tip of hollow pyramidal absorber with impedance matching allow them to reach the absorber and induce wave reflection between pyramidal absorbers [9]-[11].

In the microwave region, carbon is commonly used to coat the absorbers. Carbon is one of the semiconductors that allow a little amount of charge flow through it [12], [13]. As a microwave strikes this material, the wave becomes attenuated and weakend. The cancellation phase will take part when energy is loss due to the conversion from electromagnetic interference energy to heat energy [14]-[16]. The dielectric 
material of the absorbers are characterized by electric permittivity and magnetic permeability. The permittivity is a proportion of the material's impact on the electric field in the electromagnetic wave and permeability is a proportion of the material's impact on the magnetic component of the wave [15], [17], [18].

The attenuation of electromagnetic wave can be obtained by reflection or absorption of the received signal [19], [20]. Many studies have been done on the development of highperformance multifunctional absorbers. Jaumann absorbers and Salisbury screen are very good examples of radiation absorbers. In the literatures, Salisbury screen is a passive microwave absorber which is built by putting a thin $377 \Omega$ /square resisitive sheet $\lambda / 4$ over a metal ground plane [21], [22]. This condition is to diminish backscaterring from a flat plate at normal incidence. It is a narrow-band technique because the placement of the resisitive sheet is a function of frequency and is critical to the radiation cross section (RCS) performance [23]. It also can expand the bandwidth by adding the additional capacitive sheets, inventing Jaumann absorber [24], [25].

In previous research, only several works performed on the optimization of pyramidal microwave absorber by using slot technique. A significant finding by A.Hasnain is that the new technique of slot radial array design on pyramidal microwave absorber is proved to improve the absorption performance [26]. The absorption performance of pyramidal microwave absorber with slotted design is increased from $-10.3 \mathrm{~dB}$ to $-11.8 \mathrm{~dB}$. This challenge was then attempted to be solved in subsequent work. For example, the slotted triangle on pyramidal microwave absorber is presented in order to investigate the effect of the performance after applying the sierpinski triangle slot design. It was found that the best absorption performance is high up to $38.56 \mathrm{~dB}$ at frequency range $11 \mathrm{GHz}$ to $12 \mathrm{GHz}$ [27]. Meanwhile, A.Syahmi et.al investigated the impact of the selective point on the microwave absorber surface with un-slotted and inverted triangle slotted. The results indicate that the highest absorption performance obtain is $-20.65 \mathrm{~dB}$ which is higher than un-slotted design [23].

Numeric methods and equivalent cicuit method are the principal tools of analysis for Frequency Selective Surface (FSS) absorbers [28], [29]. Some method of optimization is also required due to the wave absorbing performance parameters. Technically, there are many shapes and arrangements in FSS method such as square, square rings, rectangular, cross dipole and arbitrarily geometry. Shape is the most sensitive parameter which can impact the RAM's reflectivity performance [30]. In addition, the absorber is independent of the polarization of an incident wave regardless of its geometrical shape [31]. FSS or slot array consists of capacitive cells, acts as a capacitor on the low frequency region but its impedance after the first resonance becomes inductive [28]. The impedance of a slot can be represented through a series RLC circuit as shown in Figure 1.

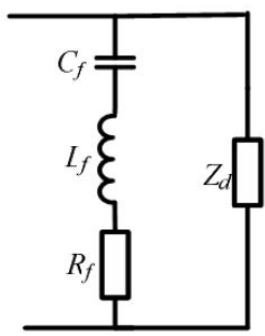

Fig. 1. Equivalent transmission-line circuit model [31]

The shape of the slot influence the values of $\mathrm{L}$ and $\mathrm{C}$ parameters. The dielectric substrate (carbon) behaves as an inductor in this work.

In theory, slot is defined as a radiating element to excite the electromagnetic wave. A slot is designed to reflect, transmit or absorb electromagnetic fields based on the frequency of field [32]. The currents flow around the slot and build up charge on one side, which causes an electric field to form across the slot. The field across the gap depends on the current flowing around the slot. By placing a slot in the path of the currents, the slots can be excited and energy radiated. An incoming wave of the plane is either transmitted (passband) or reflected (stopband), entirely or partially, depending on the feature of the nature array element.[33]. This happens as the electromagnetic wave matches the slot feature resonant frequency. The slot is thus capable of passing or blocking the electromagnetic wave of a certain frequency spectrum in free space[33]-[35]. For the rectangular slot, the resonance occurs when the length of each half-loop is a multiple of one half-wavelength. This means that a half-loop acts as a dipole. Therefore, the total length of the loop should be a multiple of one full wavelength. If a vertically polarized incoming wave strikes a vertical half-wave vertical dipole, the dipole resonates, regardless of the angle of incident [33]. However, the dipole does not resonate efficiently depending on the angles of the incident where the direction of incident is oblique to the width of the dipole. This is because the length of the dipole in the incident direction is now less than a half-wavelength.

In this article, the rectangular slot shape of the microwave absorber is proposed as a new alternative for enhancing absorption performance. By changing the material's carbon loading, the optimal performance of absorbers is achieved. The loading needed is light enough to increase the penetration of the wave during each reflection, but strong enough to absorb this part of the wave before emerged. The absorption performance can be increased by using slots in the absorber surface by preserving all other parameters including shape, material and carbon loading. Moreover, owing to the previous excellent performance has initiated this study to investigate the rectangular slotted design at different size. In this study, potential outcomes to improve the absorption performance of the hollow pyramidal microwave absorber has been considered by investigating several size of rectangular slots. The concept of slot is applied to the electromagnetic wave in order for the wave to get radiated. To excite the wave, there must be a coupling of $\lambda g / 4$ for $E$ field. However, currently there is no evidence in microwave absorber regarding to the slots theory 
and the concept of antenna slot theory is applied unto the microwave absorber due to microwave absorber concept is inversely proportional to antenna theory.

\section{Methodology}

The initial study of this project began by collecting and conducting several studies on microwave absorber and a review of the literatures of previous researchers. Basically, the commercial microwave absorbers have their standard electromagnetic properties at certain frequencies. The available commercial microwave absorbers have dimension ranging from $5.1 \mathrm{~cm}$ ( 2 inch) up to $3.7 \mathrm{~m}$ (12ft). Special cuts often made for specific applications. Table 1 shows several electromagnetic properties of commercial pyramidal microwave absorber available in the market. These commercial pyramidal absorbers are non-slot solid pyramidal shape.

TABLE I

COMPARISON ELECTROMAGNETIC PROPERTIES OF COMMERCIAL ABSORBERS AVAILABLE IN THE MARKET

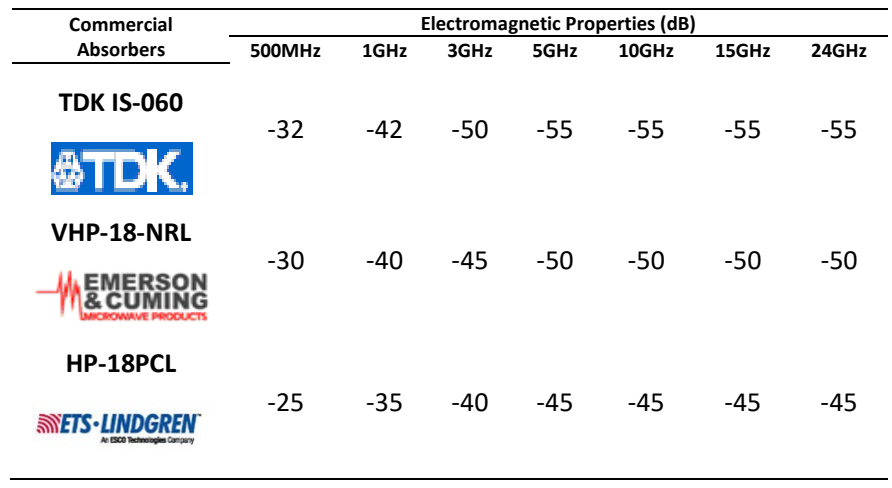

Several proposed slotted designs with different size has been considered including the shape, size and dielectric material used to meet the scope of the project. The simulation and measured performance of the proposed slotted microwave absorber has been performed by using CST simulation software and NRL arch free space measurement. The CST simulation software is used to estimate the capability performance of the designed while NRL arch free space is a method to verify the actual performance of the slotted microwave absorber design. The actual performance results obtained by each slotted designs are analysed and discussed in this paper.

\section{A. CST Simulation Modelling}

After studies on characteristics and parameters have been performed, several factors influencing the absorption performance are identified to determine the absorption performance of the proposed slotted design. Several design of single rectangular slot on hollow pyramidal microwave absorber are designed by using CST simulation in order to predict the preliminary results of the absorption performance. Each design is applied with different size of the slot design, however the value of the dielectric constant applied is still the same. The desired frequency range is set within 1 to $12 \mathrm{GHz}$ to operate the simulation performance of the designed absorber.
The reflection coefficient simulated results obtained were analysed for the absorption performance indicator .

\section{B. Fabrication Process}

Fig. 2 shows several designs of single rectangular slot on hollow pyramidal microwave absorber that have been proposed in this project. There are three different size of rectangular slot design in this study namely small, medium and big slot size. The rectangular shape of the slot has been chosen based on the desired frequency range of $1 \mathrm{GHz}$ to $12 \mathrm{GHz}$. In this project, the selected frequency of $2 \mathrm{GHz}, 4 \mathrm{GHz}$ and $6 \mathrm{GHz}$ are chosen to determine the size of the slots. The higher the frequency the lower the slot size. The size of rectangular slots can be calculated by using equation (1) and (2) as following :

$$
\begin{aligned}
& \lambda=\frac{c}{f} \\
& \mathrm{~L}=\frac{\lambda}{2}
\end{aligned}
$$

Where, the height of the slot must be $\mathrm{H}<<\boldsymbol{\lambda}$.

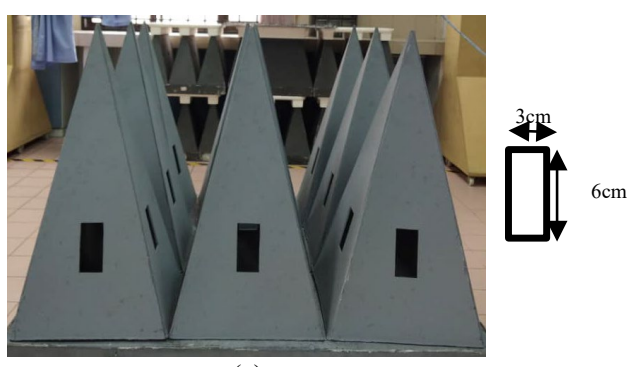

(a)

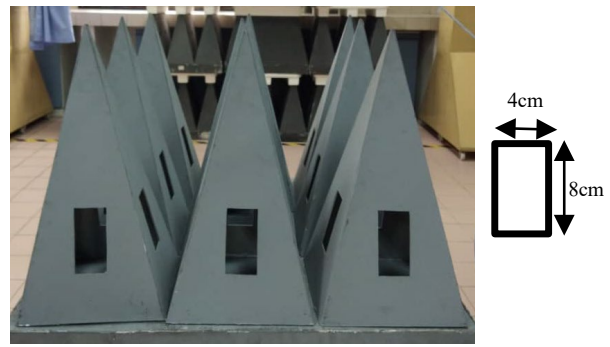

(b)

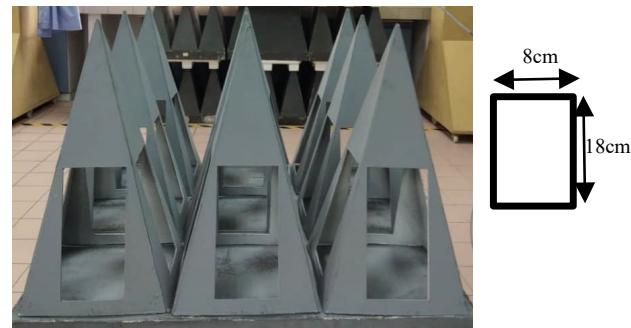

(c)

Fig. 2. The different size of single rectangular slot design on hollow pyramidal microwave absorber (a) small slot (b) medium slot (c) big slot

The fabrication process started with preparing the standard commercial pyramidal size microwave absorber. The material 
to form the hollow pyramidal microwave absorber is created from cardboards. The next process is implementing the proposed rectangular slot on the four sides of hollow pyramidal microwave absorber. The slot designs are divided into three size which is small, medium and big slot size respectively. For absorbing material, the preparation of the appropriate material ratio is taken into account. In this study, carbon which is derived from biomass material is going to be used as a coating material or radiation absorbing material (RAM). The powder activated carbon (PAC) is blended together with water and painted to form a coating paint to coat the surface of the pyramidal absorber.

\section{NRL Arch Free Space Measurement}

Last step is to measure the absorber designs by using the measurement of NRL Arch free space method. Ayat perlu diubah. The standard measurement set up is shown in Fig. 3 to verify the absorption performance of the proposed design. The calibration measurement set up for free space arch method has a very simple procedure by placing the plate on the centre with transmitting and receiving signal wave from horn antenna at normal incidence [36]-[38]. The transmitter antenna is connected to the signal generator and sends microwave energy to the material being tested while the receiving antenna is connected to the signal detector and measures the excess energy after reflection.

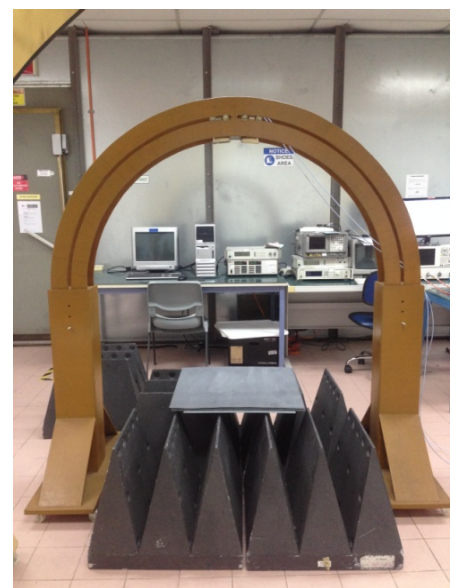

Fig. 3. Measurement set up by using NRL arch free space method

\section{RESULTS AND DISCUSSION}

The performance of different size of slotted design is discsussed in this section. There are three different size of single rectangular slot on hollow pyramidal absorber. They are compared and analysed in term of their absorption performance towards four frequency band which are L, S, C and X-band. The slotted designed are labelled with the name of small, medium and big slot size respectively as shown in Fig. 1. Fig. 3 shows the comparison of absorption performance obtained by all designs.

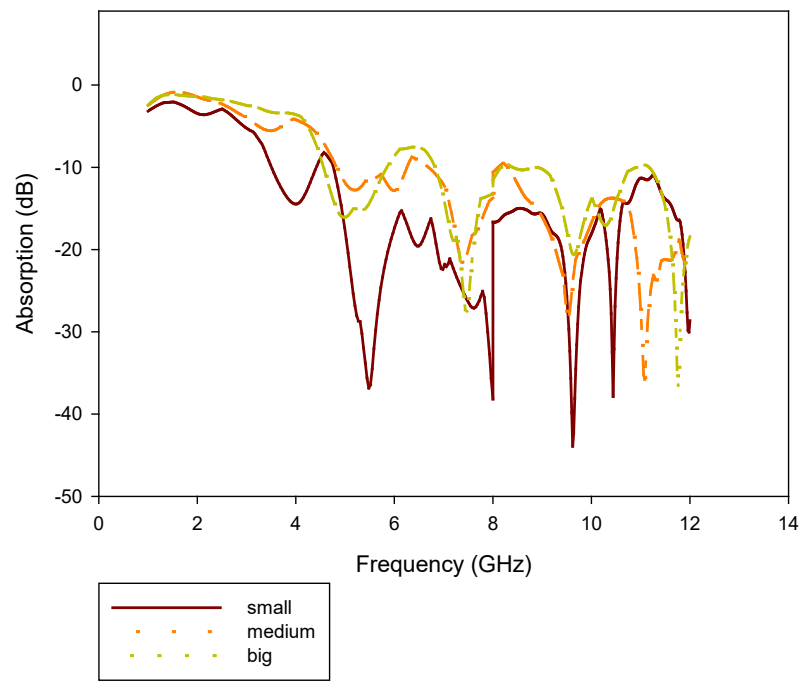

Fig. 4. The measurement results of small slot, medium slot and big slot size

Fig. 4 shows the results obtained from the measurement analysis of the small slot, medium slot and big slot. The comparison of the different slot size are analysed to examine the effect of absorption performance towards frequency band application. As shown in the graph, the performance achieved by a small slot size design is better than medium and big slot size at all frequency band. The highest absorption performance obtained by small slot at the entire frequency range of $1 \mathrm{GHz}$ to $12 \mathrm{GHz}$, is $-43.89 \mathrm{~dB}$ at $9.62 \mathrm{GHz}$

TABLE II

MINIMUM, MAXIMUM AND AVERAGE ABSORPTION PERFORMANCE OF SMALL SLOT, MEDIUM SLOT AND BIG SLOT SIZE

\begin{tabular}{|c|c|c|c|c|c|}
\hline \multirow[t]{2}{*}{ Band } & \multirow{2}{*}{$\begin{array}{l}\text { Frequency } \\
(\mathrm{GHz})\end{array}$} & \multirow[t]{2}{*}{ Design } & \multicolumn{3}{|c|}{ Absorption (dB) } \\
\hline & & & Min & Max & Average \\
\hline \multirow[t]{3}{*}{ L-band } & \multirow[t]{3}{*}{$1-2$} & Small & -2.06 & -3.39 & -3.02 \\
\hline & & Medium & -0.84 & -2.46 & -2.28 \\
\hline & & Big & -1.12 & -2.38 & -2.19 \\
\hline \multirow[t]{3}{*}{ S-band } & \multirow[t]{3}{*}{$2-4$} & Small & -2.91 & -14.44 & -3.58 \\
\hline & & Medium & -1.92 & -5.54 & -1.60 \\
\hline & & Big & -1.36 & -3.53 & -1.37 \\
\hline \multirow[t]{3}{*}{ C-band } & \multirow[t]{3}{*}{$4-8$} & Small & -8.20 & -38.16 & -14.38 \\
\hline & & Medium & -4.22 & -21.58 & -4.31 \\
\hline & & Big & -3.59 & -27.47 & -3.66 \\
\hline \multirow[t]{3}{*}{$\mathrm{X}$-band } & \multirow[t]{3}{*}{$8-12$} & Small & -10.89 & -43.89 & -16.64 \\
\hline & & Medium & -9.48 & -35.76 & -13.69 \\
\hline & & Big & -9.41 & -36.46 & -13.14 \\
\hline
\end{tabular}




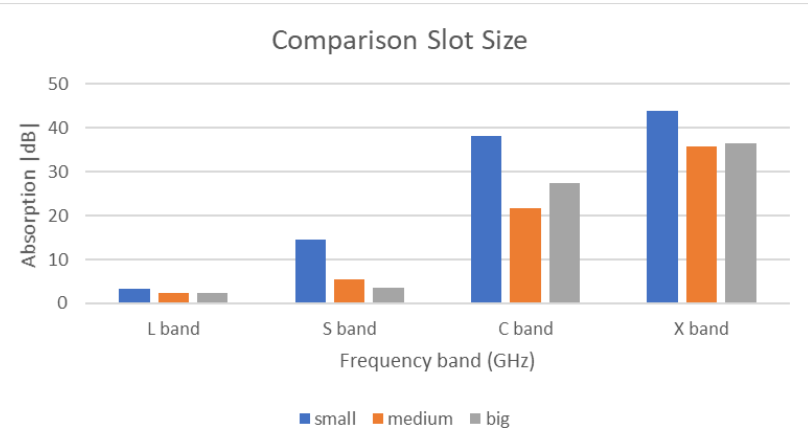

Fig. 5. Maximum absorption performance of small slot, medium slot and big slot size

Table 2 compares the data for minimum, maximum and average absorption performance obtained by small slot, medium slot and big slot size. While, Figure 5 shows the maximum absorption performance achieved by three different size of slot designs are compared and analysed by using the bar chart . It can be seen from the Figure 2, the average absorption performance of small slot size is $-3.02 \mathrm{~dB}$ at L-band, $-3.58 \mathrm{~dB}$ at S-band, $-14.38 \mathrm{~dB}$ at C-band and $-16.64 \mathrm{~dB}$ at X-band, which are better than medium and big slot size. The medium slot size obtained higher average absorption performance than big slot size at all frequency band which are $-2.28 \mathrm{~dB}$ at L-band, $-1.60 \mathrm{~dB}$ at S-band, $-4.31 \mathrm{~dB}$ and at C-band and $-13.69 \mathrm{~dB}$ at X-band. Meanwhile, the average absorption performance of big slot size is $-2.19 \mathrm{~dB}$ at L-band, $-1.37 \mathrm{~dB}$ at S-band, $-3.66 \mathrm{~dB}$ at C-band and $-13.14 \mathrm{~dB}$ at $\mathrm{X}$-band. Among the three candidates small slot size has the highest average absorption performance followed by medium and big slot size.

The analysed data for minimum absorption performance obtained by small slot size shows a better minimum absorption performance than medium and big slot size at all frequency band. If a narrow length of the slot is cut in a large flat sheet, it will absorb in a manner resembling a particular radiation with a dipole radiation of the same dimensions as the slot. Consequently, the minimum absorption of small slot design obtained the highest performance than medium and big slot size which are $-2.06 \mathrm{~dB}$ at L-band, $-2.91 \mathrm{~dB}$ at S-band, $-8.20 \mathrm{~dB}$ at Cband and $-16.64 \mathrm{~dB}$ at $\mathrm{X}$ band. Apart of that, the medium slot size contribute better minimum absorption performance than big slot size at S-band, C-band and X-band which is $-1.92 \mathrm{~dB}$, $4.22 \mathrm{~dB}$ and $-9.48 \mathrm{~dB}$ respectively except at L-band which is $0.84 \mathrm{~dB}$ and $-1.12 \mathrm{~dB}$ respectively. Meanwhile, the big slot size obtained the lowest absorption performance at S-band, C-band and $X$ band which are $-1.36 \mathrm{~dB},-3.59 \mathrm{~dB}$ and $-9.41 \mathrm{~dB}$ respectively.

The bar chart in Fig. 4 indicates that small slot size achieved the highest maximum absorption performance at all frequency band. The maximum absorption performance obtained by small slot size is $-3.39 \mathrm{~dB}$ at L-band, $-14.44 \mathrm{~dB}$ at S-band, $-38.16 \mathrm{~dB}$ at C-band and $-43.89 \mathrm{~dB}$ at X-band which indicated better performance than medium slot and big slot. The medium slot size obtained higher maximum absorption than big slot size at low frequency band which are $-2.46 \mathrm{~dB}$ at L-band and $-5.54 \mathrm{~dB}$ at $\mathrm{S}$-band respectively. Meanwhile, the big slot size provide better maximum absorption than medium slot size at high frequency band of $-27.47 \mathrm{~dB}$ at C-band and $-36.46 \mathrm{~dB}$ at X-band. The result conclusively prove that the smaller the size of the slot, the better the absorption performance is, at certain frequency band. From this results, it is indicated that the slot size play a significant role to improve the absorption performance towards the frequency band. The slot length of small, medium and big size is designed based on frequency $6 \mathrm{GHz}, 4 \mathrm{GHz}$ and $2 \mathrm{GHz}$ respectively. Based on the results, the absorption performance of different slot length occurred at the designed frequency. The different size of the slot width will influence the different bandwith performance. As shown in Fig. 4 , the small slot with narrow width produce a narrow bandwidth at frequency band. This research proves that excellent absorption performance is achieved by small slot size across all frequency band as compared to medium and big slot size. It can be concluded that the small slot that has a narrow slot length facing the normal incident wave provide a better efficiency of radiation absorption. This study demonstrated that the wavelength of the slot can be adjusted by varying the slot length in order to improve the absorption performance.

\section{CONCLUSION}

This paper has presented an account of slot size play an important role to improve the absorption performance within the frequency range of $1 \mathrm{GHz}$ to $12 \mathrm{GHz}$. In this investigation, the effect of different slot size towards absorption performance at certain band applications are experimentally verified. The most obvious finding to emerge from this study is that small slot size contribute better performance at L-band, S-band, Cband and X-band.

\section{ACKNOWLEDGMENT}

The authors would like to thank the RMI, Universiti Teknologi MARA Cawangan Pulau Pinang, Malaysia for providing the financial support and equipment assistant for this work through the Research Grant 600-IRMI/FRGS 5/3 (193/2019) and FRGS/1/2018/TK1 0/UITM/02/20.

\section{REFERENCES}

[1] M. M. Dawoud, "High Frequency Radiation and Human Exposure," in Proceedings of the International Conference on Non-Ionizing Radiation at UNITEN (ICNIR 2003) Electromagnetic Fields and Our Health, 2003, no. October, pp. 1-7.

[2] F. Marra, J. Lecini, A. Tamburrano, L. Pisu, and M. S. Sarto, "Electromagnetic wave absorption and structural properties of wideband absorber made of graphene-printed glass-fibre composite," Sci. Rep., vol. 8, no. 1, pp. 1-9, 2018.

[3] E. Kivrak, K. Yurt, A. Kaplan, I. Alkan, and G. Altun, "Effects of electromagnetic fields exposure on the antioxidant defense system," J. Microsc. Ultrastruct., vol. 5, no. 4, p. 167, 2017.

[4] D. A. Vinnik et al., "Magnetic and Structural Properties of Barium Hexaferrite BaFe12O19 from Various Growth Techniques," Materials (Basel)., vol. 10, no. 578, pp. 2-11, 2017.

[5] Arthur von Hippel, "Theory and Applications of RF/Microwave Absorbers," 2012.

[6] A. N. Yusoff, M. H. Abdullah, S. H. Ahmad, S. F. Jusoh, and A. A. Mansor, "Electromagnetic and absorption properties of some microwave absorbers," J. Appl. Phys., vol. 92, no. 2, pp. 876-882, 2002.

[7] L. M. Kasim, N. M. Noor, H. Abdullah, A. Ahmad, and N. Mohamat, 
"A Study of Electromagnetic Absorption Performance of Modern Biomass Wall Tile," Int. J. Electr. Electron. Eng. Telecommun. A, vol. 9, no. 6, pp. 9-13, 2020.

[8] H. Nornikman, P. Soh, and A. Azremi, "Performance of Different Polygonal Microwave Absorber Designs Using Novel Material," in International Symposium on Antennas and Propagation, 2009, pp. 1151-1154.

[9] L. Y. Seng et al., "Enhanced microwave absorption of rice huskbased pyramidal microwave absorber with different lossy base layer," IET Microwaves, Antennas Propag., vol. 14, no. 3, pp. 215-222, 2019.

[10] S. I. Orakwue and I. P. Onu, "Pyramidal Microwave Absorber Design for Anechoic Chamber in the Microwave Frequency Range of $1 \mathrm{GHz}$ to 10GHz," Eur. J. Eng. Res. Sci., vol. 4, no. 10, pp. 1-3, 2019.

[11] H. Nornikman, P. J. Soh, A. A. H. Azremi, and M. S. Anuar, "Performance simulation of pyramidal and wedge microwave absorbers," in Proceedings - 2009 3rd Asia International Conference on Modelling and Simulation, AMS 2009, 2009, pp. 649-654.

[12] H. Nornikman, "Investigation of an agricultural waste as an alternative material for microwave absorbers Investigation of an Agricultural Waste as an Alternative Material for Microwave Absorbers," in Progress In Electromagnetics Research Symposium Proceedings, 2009, no. 8, pp. 1287-1291.

[13] I. M. Ibrahim, M. N. Husain, S. Se, and A. Shaaban, "The effect of the carbon to the S11 measurement on the pyramidal microwave absorbers," in Wireless Technology and Applications (ISWTA), 2011 IEEE Symposium, 2011, no. 2, pp. 2-6.

[14] S. S. Pattanayak, "A Review on Microwave Absorber Using Agricultural Residues," in 7th Indian Young Geotechnical Engineers Conference, 2019, no. February, pp. 4-8.

[15] C. Ellison, M. S. McKeown, S. Trabelsi, and D. Boldor, "Dielectric properties of biomass/biochar mixtures at microwave frequencies," Energies, vol. 10, no. 4, pp. 1-11, 2017, doi: 10.3390/en10040502.

[16] F. Paquin, J. Rivnay, A. Salleo, N. Stingelin, and C. Silva, "Review on the Electromagnetic Interference Shielding Materials fab- ricated by Iron Ingredients," Nanoscale Adv., vol. 3, no. 4, pp. 10715-10722, 2019.

[17] N. A. Zulkifli et al., "Analysis of Dielectric Properties on Agricultural Waste for Microwave Communication Application," in MATEC Web of Conferences, 2017, vol. 140, pp. 1-5.

[18] S. N. Ab Jabal, Y. B. Seok, and W. F. Hoon, "Carbon composition, surface porosities and dielectric properties of coconut shell powder and coconut shell activated carbon composites," ARPN J. Eng. Appl. Sci., vol. 11, no. 6, pp. 3832-3837, 2016.

[19] N. F. N. Yah, H. A. Rahim, Y. S. Lee, F. H. Wee, and H. H. Zainal, "Electromagnetic wave absorption properties of novel green composites coconut fiber coir and charcoal powder over X-band frequency for electromagnetic wave absorbing applications," $A d v$. Electromagn., vol. 7, no. 1, pp. 13-18, 2018.

[20] F. M. Idris, M. Hashim, Z. Abbas, I. Ismail, R. Nazlan, and I. R. Ibrahim, "Recent developments of smart electromagnetic absorbers based polymer-composites at gigahertz frequencies," J. Magn. Magn. Mater., vol. 405, no. July 2018, pp. 197-208, 2016.

[21] F. Che Seman and R. Cahill, "Frequency selective surfaces based planar microwave absorber," in Progress in Electromagnetics Research Symposium, 2012, vol. 1, pp. 906-909.

[22] F. C. Seman, R. Cahill, and V. F. Fusco, "Electronically Tunable Liquid Crystal Based Salisbury Screen Microwave Absorber," in Loughborough Antennas \& Propagation Conference, 2009, no. November, pp. 93-96.

[23] A. Syahmi et al., "Radiation Cross Section Characteristics for Isosceles Slotted Triangle on Hollow Pyramidal Absorber," in 2018 IEEE International Conference on Automatic Control and Intelligent Systems (I2CACIS), 2018, pp. 1-4.

[24] A. Kazemzadeh and A. Karlsson, "On the Absorption Mechanism of Ultra Thin Absorbers," IEEE Trans. Anttenna Propag., vol. 58, no. 10, pp. 3310-3315, 2010.

[25] A. Cheldavi, "Optimum Design of N Sheet Capacitive Jaumann Absorber Using Genetic Algorithm," IEICE Trans. Fundam., vol. E82-A, no. 4, pp. 704-706, 1999.

[26] R. R. R. Dessai and H. G. Virani, "ER ER," vol. 2, no. 3, pp. 2-5, 2013.

[27] A. S. Yusof et al., "Slotted triangle on hollow pyramidal microwave absorber characteristics," in Proceedings - 6th IEEE International Conference on Control System, Computing and Engineering,
ICCSCE 2016, 2017, no. November, pp. 563-568.

[28] F. Costa, A. Monorchio, and G. Manara, "Analysis and design of ultra thin electromagnetic absorbers comprising resistively loaded high impedance surfaces," IEEE Trans. Antennas Propag., vol. 58, no. 5, pp. 1551-1558, 2010.

[29] Y. N. Kazantsev et al., "Broadening of operating frequency band of magnetic-type radio absorbers by FSS incorporation," IEEE Trans. Antennas Propag., vol. 58, no. 4, pp. 1227-1235, 2010, doi: 10.1109/TAP.2010.2041316.

[30] M. W. B. Silva and L. C. Kretly, "A new concept of RAM-Radiation Absorbent Material: Applying corrugated surfaces to improve reflectivity," in SBMO/IEEE MTT-S International Microwave and Optoelectronics Conference Proceedings, 2011, no. 11, pp. 556-560.

[31] L. Sun, H. Cheng, Y. Zhou, and J. Wang, "Broadband metamaterial absorber based on coupling resistive frequency selective surface," in Optics Express, 2012, vol. 20, no. 4, pp. 4675-4680.

[32] A. A. Olinert, "The Impedance Properties of Narrow Radiating Slots in the Broad Face of Rectangular Waveguide," IRE Trans. Antennas Propag., vol. 4, no. 1, pp. 1-5, 1957.

[33] T. Wu, A. Consulting, and E. Shielding, Frequency Selective Surfaces, no. 5. 2016.

[34] R. S. Anwar, L. Mao, and H. Ning, "Frequency Selective Surfaces: A Review," J. Appl. Sci., vol. 8, no. 1689, pp. 1-46, 2018.

[35] R. Panwar and J. R. Lee, Progress in frequency selective surfacebased smart electromagnetic structures: A critical review, no. 3 . Elsevier Masson SAS, 2017.

[36] M. S. Mezan, M. F. A. Malek, M. S. Jusoh, F. S. Abdullah, and N. A. M. Affendi, "Reflection loss performance and performance assessment of pyramidal microwave absorber using agriculture waste," in Progress in Electromagnetics Research Symposium, 2014, no. August, pp. 142-145.

[37] N. M. F. and M. N. T. N Mohamat Kasim, H Abdullah@Idris, M F A B Ali Mokhtar, A Ahmad, N Mohamad Noor, L Mohd Kasim, N A Ismail, "Corrugated bamboo roofing design of microwave absorber for reflection absorption Corrugated bamboo roofing design of microwave absorber for reflection absorption," in Journal of Physics, 2019, pp. 1-7.

[38] A. Ahmad, H. Bin Abdullah, L. M. Kasim, N. A. Ismail, and N. M. Noor, "A Study of Carbon Coated Flat Stick Bamboo Microwave Absorber Performance," Int. J. Electr. Electron. Eng. Telecommun. $A$, vol. 9, no. 5, pp. 8-13, 2020.

Mas Izzati Binti Fazin was born in Kedah, Malaysia, on August 10, 1994. She received her diploma in Electronic Engineering and B.Sc. degree in Electrical and Electronic Engineering in class 2015 and 2018 respectively, from University of Technology MARA (UiTM) Malaysia. Currently, she is pursuing her $\mathrm{Ph} . \mathrm{D}$ in communication engineering at the same university. And working as Research Asisstant at the Microwave and Radiation Laboratory, University of Technology Mara (UiTM) Pulau Pinang. Her current research focusses on biomass microwave absorber, radio frequency and electromagnetic.

Dr. Ahmad Rashidy Bin Razali received his B.Eng (Hons) degree in Electronic Engineering from University Tenaga Nasional, Selangor, Malaysia in 2002. He obtained his M.Sc degree in Mobile and Satellite Communication Engineering from University of Surrey, United Kingdom in 2004 and in 2012, he received his Ph.D in Communication Engineering from University of Queensland, Australia. He is currently an Associate Professor at University Technology Mara, Pulau Pinang Malaysia. He is a Professional Engineer recognized by Board of Engineer Malaysia (BEM). His research interests include in antenna, microwave and communication system.

Dr. Hasnain Bin Abdullah@Idris received his B.Sc degree in Electrical Engineering and M.Sc degree in Communication 
Engineering from University of Technology Malaysia (UTM) Skudai, Malaysia, in 1996 and 2002, respectively. In 2019, he obtained his Ph.D in Electrical Engineering from University of Technology Mara, Shah Alam, Malaysia. He is currently a lecturer in University of Technology Mara, Pulau Pinang, Malaysia. His current research interests include antenna and propagation, radio frequency, microwave and communication system. Presently, he is a leading design and development of microwave absorber in UiTM Pulau Pinang.

Dr. Mohd Nasir Bin Taib, PhD, is a Professor at the Faculty of Electrical Engineering, University of Technology Mara (UiTM). He received his degree in Electrical Engineering from University of Tasmania, Australia, M.Sc. in Control Engineering from Sheffield University, UK and the PhD degree in Instrumentation from University of Manchester, UK. He heads The Advanced Signal Processing research group (ASPRG) which is very active in research and applications of control system, biomedical engineering, energy studies, optical fiber sensors and nonlinear system. He has published more than 200 technical papers, locally and international level. Currently, he is the Chair of the IEEE Malaysia and has been awarded twice as the Best Researcher in UiTM (in 2003 and 2005) and received many national and international awards, and patents for his research and inventions.

Norhayati Mohamad Noor was born in Kedah, Malaysia in 1975. She received her Bachelor of Electrical Engineering and Master of Electrical Engineering from Universiti Teknologi Malaysia, Johor in 1998 and 2002 respectively. In 2000, she joined the Faculty of Electrical Engineering, Universiti Teknologi MARA, Pulau Pinang as a Lecturer and became a Senior Lecturer since 2010. Her current research interests include Mobile communication and telecommunication.

Nazirah Mohamat Kasim was born in Perak, Malaysia in 1976. She received her Dip.Eng. in electrical engineering and the B.Eng. degree (with honors) in electrical engineering from the Universiti Teknologi Mara, Malaysia, in 1998 and 2001 respectively. She completed her study in M.Sc. degree in microelectronics from the Universiti Kebangsaan Malaysia, Malaysia, in 2005. She joined the Faculty of Electrical Engineering, Universiti Teknologi Mara, Pulau Pinang, Malaysia, in 2001, where she is currently senior lecturer since 2011. Her research interests cover microwave engineering, telecommunication and microelectronic.

Azizah Ahmad was born in Penang, Malaysia in 1982. She received her Diploma and B.Eng. Hons in Electrical and Electronics Engineering from Universiti Teknologi MARA in 2003 (UiTM Pulau Pinang) and 2006 (UiTM Shah Alam) respectively and M.Sc. in Electronic System Design Engineering from Universiti Sains Malaysia in 2008. In 2009, she joined the Faculty of Electrical Engineering, Universiti Teknologi MARA, Pulau Pinang as a Lecturer and became a Senior Lecturer since 2016. Her current research interests include microwave absorber, software engineering and image processing.

Linda M. Kasim was born in Perlis, Malaysia in 1976. She received her Diploma in Electronics and B.Eng. Hons. Electronics (Microelectronics) from Universiti Teknologi
Malaysia, Johor in 1997 and 1999 respectively. She completed her study in M.Sc. in electronic system design engineering from Universiti Sains Malaysia in 2005. In 2001, she joined the Faculty of Electrical Engineering, Universiti Teknologi MARA, Pulau Pinang as a Lecturer and became a Senior Lecturer since 2012. Her current research interests include electromagnetic absorbing material, microwave absorber and power electronics.

Noor Azila Ismail was born in Kedah, Malaysia, in 1976. She received the B.Eng. (Hons) in electrical engineering and the M.Eng. Electrical Energy and Power System in electrical engineering from the Universiti Malaya, Malaysia in 2000 and 2006 respectively. In 2006, she joined the Faculty of Electrical Engineering, Universiti Teknologi MARA. Cawangan Pulau Pinang, as a Lecturer, and in 2011 became a Senior Lecturer. Her current research interests include microwave, communication, power electronics and drives. 